\title{
All entangled states are useful for information processing
}

\author{
Lluís Masanes \\ School of Mathematics, University of Bristol, Bristol BS8 1TW, U.K.
}

(Dated: October 8, 2018)

\begin{abstract}
The question whether all entangled states can be used as a nonclassical resource has remained open so far. Here we provide a conclusive answer to this problem for the case of systems shared by two parties. We show that any entangled state $\sigma$ can enhance the teleportation power of some other state. This holds even if the state $\sigma$ is bound entangled.
\end{abstract}

It is widely said that "entanglement is a physical resource", but does this mean that all entangled states are resources? The definition of entangled state is made in terms of the physical resources needed for the preparation of the state: a state is said to be entangled if it cannot be prepared by local (quantum) operations and classical communication (LOCC). Mathematically, entangled states are the ones which are not separable [1]. This definition in terms of preparation tells us nothing about the resources that can be extracted from an entangled state. Until now, the existence of entangled states with no extractable "quantum resources" has not been ruled out. In this letter we prove that in the bipartite scenario such states do not exist.

One of the central ideas in quantum information theory is quantum teleportation [3]. This procedure allows one to use a bipartite quantum system in an entangled state as a quantum channel. Remarkably, this has been experimentally implemented [4]. If for a particular state the quality of its corresponding teleportation channel is too low, one can sometimes do better by allowing the procedure to fail with some probability, and the sender/receiver know when this happens. This scenario is called conclusive teleportation, and was introduced in [5]. The result of the present letter can be read as follows: any entangled state can enhance the conclusive teleportation fidelity of another state. But before going into this, let us recall some results suggesting the uselessness of some entangled states.

In 1989 Werner presented examples of bipartite entangled states whose outcomes according to any local measurement can be simulated by classical correlations [2], see also [6]. In 1998 the Horodecki family proved the existence of bound entanglement, that is, entangled states from which pure-state entanglement cannot be obtained by LOCC, even with an arbitrarily large supply of copies of the state [7]. They also showed that with bound entangled states teleportation cannot be performed better than through a classical channel, even if one allows for conclusive teleportation [8].

There are also results showing that some bound en- tangled states are useful for several tasks [9-11], but none of them has been proven to hold in general. In particular, in [12], it is presented a specific bound entangled state capable of enhancing the fidelity of conclusive teleportation of an other state. In the present letter this result is generalized to all bound-entangled states.

With the original teleportation protocol [3], two parties sharing a maximally-entangled state

$$
\left|\phi_{d}\right\rangle=\frac{1}{\sqrt{d}} \sum_{s=1}^{d}|s\rangle \otimes|s\rangle,
$$

can transmit an unknown arbitrary quantum state $|\psi\rangle \in \mathbb{C}^{d}$ by LOCC. If instead of $\left|\phi_{d}\right\rangle$ the two parties share a noisy entangled state $\rho$, the teleportation channel can be imperfect. One can quantify the quality of a channel $\Gamma$ with the average fidelity of the output states with the input states

$$
F_{d}(\Gamma)=\int_{\mathbb{C}^{d}} d \psi \operatorname{tr}[\Gamma(\psi) \psi]
$$

where here and in the rest of the paper $\psi=|\psi\rangle\langle\psi|$. Note that when the shared state is not $\phi_{d}$, the optimal teleportation protocol needs not be the same as the (standard) one for $\phi_{d}$. The optimal conclusive teleportation fidelity achievable with a given state $\rho$ is considered in what follows.

Given an arbitrary bipartite state $\rho$ (acting on $\mathcal{H}_{A} \otimes \mathcal{H}_{B}$ ) we consider the $\mathbb{C}^{d} \otimes \mathbb{C}^{d}$ states $\tilde{\rho}$ that can be obtained from $\rho$ by LOCC with some probability. This probability can be arbitrarily small as long as it is nonzero. This class of transformations is called stochastic-LOCC (SLOCC). Each of these states $\tilde{\rho}$ is the normalized output of a separable (not necessarily trace-preserving) completely-positive map [13] with $\rho$ as input,

$$
\tilde{\rho}=\frac{\Omega(\rho)}{\operatorname{tr} \Omega(\rho)} .
$$

Separable maps (SEP) are the ones that can be written as

$$
\Omega(\rho)=\sum_{k} A_{k} \otimes B_{k} \rho A_{k}^{\dagger} \otimes B_{k}^{\dagger} .
$$


In our case, these matrices have the form $A_{k}: \mathcal{H}_{A} \rightarrow$ $\mathbb{C}^{d}$ and $B_{k}: \mathcal{H}_{B} \rightarrow \mathbb{C}^{d}$. The following quantity plays a central role in our discussion. Denote by $E_{d}(\rho)$ the largest overlap with the maximally-entangled state $\phi_{d}$ that a state $\tilde{\rho}$ obtainable from $\rho$ by SLOCC can achieve:

$$
E_{d}(\rho):=\sup _{\Omega \in \mathrm{SEP}} \frac{\operatorname{tr}\left[\Omega(\rho) \phi_{d}\right]}{\operatorname{tr}[\Omega(\rho)]},
$$

where the supremum is taken over all maps of the form (4) for which $\operatorname{tr}[\Omega(\rho)]>0$.

By definition, the quantity $E_{d}(\rho)$ is nonincreasing under SLOCC processing of $\rho$, and thus, an entanglement monotone [14]. One can interpret $E_{d}$ in the context of single-copy entanglement distillation [8], as the probability that $\rho$ "looks" like the maximallyentangled state $\phi_{d}$ after the optimal SLOCC transformation. There is also a relation between $E_{d}$ and teleportation. Denote by $F_{d}(\rho)$ the optimal fidelity of conclusive teleportation that can be achieved with a given state $\rho$. Notice that $d$ is the dimension of the Hilbert space associated to the teleportation channel, which has nothing to do with $\mathcal{H}_{A} \otimes \mathcal{H}_{B}$, where $\rho$ acts. It is proven in [8] that the optimal fidelity of conclusive teleportation is

$$
F_{d}(\rho)=\frac{1+d E_{d}(\rho)}{1+d} .
$$

Hence, we can also interpret $E_{d}(\rho)$ as the teleportation power of $\rho$.

The reason for writing $E_{d}(\rho)$ as a supremum instead of a maximum is because, for some states $\rho$, the set of values $\left\{\operatorname{tr}\left[\tilde{\rho} \phi_{d}\right]: \rho\right.$ can be transformed into $\tilde{\rho}$ by SLOCC $\}$ does not have a maximum. In such cases, the probability of obtaining $\tilde{\rho}$ from $\rho$ goes to zero as $\operatorname{tr}\left[\tilde{\rho} \phi_{d}\right]$ goes to $E_{d}(\rho)$. This phenomenon is called quasi-distillation in the case $E_{2}(\rho)=1$, and is considered in $[8,12]$. In [15] it is shown that if $E_{d}(\rho)>1 / d$ then $\rho$ is distillable. In complement, $E_{d}(\rho) \geq 1 / d$ holds for any $\rho$, because the state $|1\rangle \otimes|1\rangle$ can be prepared locally and its fidelity with $\phi_{d}$ is $1 / d$. Therefore, the range of $E_{d}$ is $[1 / d, 1]$. In what follows, the central result of this letter is stated.

Theorem. A bipartite state $\sigma$ is entangled if, and only if, for all $d \geq 2$ and $\lambda \in[1 / d, 1)$, there exists a bipartite state $\rho$ such that $E_{d}(\rho) \leq \lambda$ and $E_{d}(\rho \otimes \sigma)>$ $\lambda$.

In other words, any entangled state $\sigma$ is capable of increasing the fidelity with $\phi_{d}$ of another state $\rho$. Even if $\rho$ has initial fidelity arbitrarily close to 1 . In terms of teleportation the interpretation of the theorem is also clear. We can chose the threshold fidelity $\lambda$ above which we get satisfactory teleportation according to our needs (for example, this threshold could be the teleportation fidelity through a perfect classical channel). Now, we can consider the set of states $\rho$ whose fidelity of conclusive teleportation is upper bounded by the chosen threshold $\lambda$. If a state $\sigma$ is entangled, no matter how weakly entangled it is, it can help another state $\rho$ to achieve a fidelity strictly larger than the threshold $\lambda$. In particular, all bipartite bound entangled states are capable of enhancing the teleportation power of other states. Recall that bound entanglement alone cannot teleport at all [8], hence this result is quite remarkable.

Proof of the theorem. If $\sigma$ is a separable state [1] we have that $E_{d}(\rho \otimes \sigma)=E_{d}(\rho)$ for any $\rho$. This holds because separable states can be created by LOCC, and $E_{d}$ is nonincreasing under LOCC. Let us prove the other direction of the equivalence.

From now on $\sigma$ is an arbitrary entangled state acting on $\mathcal{H}_{A_{1}} \otimes \mathcal{H}_{B_{1}}$. Also, $d$ and $\lambda$ are fixed to some arbitrary values in their allowed ranges, $d \geq 2$ and $\lambda \in[1 / d, 1)$. We have to show that there always exists a state $\rho$ such that $E_{d}(\rho) \leq \lambda$ and $E_{d}(\rho \otimes \sigma)>\lambda$. We fix $\rho$ to act on $\left[\mathcal{H}_{A_{2}} \otimes \mathcal{H}_{A_{3}}\right] \otimes\left[\mathcal{H}_{B_{2}} \otimes \mathcal{H}_{B_{3}}\right]$, where $\mathcal{H}_{A_{2}}=\mathcal{H}_{A_{1}}, \mathcal{H}_{B_{2}}=\mathcal{H}_{B_{1}}$, and $\mathcal{H}_{A_{3}}=\mathcal{H}_{B_{3}}=\mathbb{C}^{d}$. From (5) one can see that $E_{d}(\rho)=E_{d}(\tau \rho)$ for any number $\tau>0$. Then, for convenience, in the rest of the proof $\rho$ is allowed to be not normalized. The only constraints on $\rho$ for being a state are $\rho \succeq 0$ and $\rho \neq 0$ (we denote the operator inequality by $\succeq$ and the real-number inequality by $\geq$ ). Condition $\rho \succeq 0$ is equivalent to

$$
\operatorname{tr}[\rho P] \geq 0 \quad \forall P: P \succeq 0,
$$

which defines a convex cone [16].

Given a finite list of pairs of positive numbers $\left(x_{1}, y_{1}\right), \ldots\left(x_{n}, y_{n}\right)$ the following inequality can be proven by induction:

$$
\frac{x_{1}+\cdots+x_{n}}{y_{1}+\cdots+y_{n}} \leq \max _{i} \frac{x_{i}}{y_{i}} .
$$

Using it, one can see that in expression (5) the supremum is always achieved by a map $\Omega$ with only one term:

$$
E_{d}(\rho)=\sup _{A, B} \frac{\operatorname{tr}\left[A \otimes B \rho A^{\dagger} \otimes B^{\dagger} \phi_{d}\right]}{\operatorname{tr}\left[A \otimes B \rho A^{\dagger} \otimes B^{\dagger}\right]}
$$

where $A, B$ are matrices of the form $A:\left[\mathcal{H}_{A_{1}} \otimes \mathbb{C}^{d}\right] \rightarrow$ $\mathbb{C}^{d}, B:\left[\mathcal{H}_{B_{1}} \otimes \mathbb{C}^{d}\right] \rightarrow \mathbb{C}^{d}$. With (9) we can characterize the set of matrices $\rho$ satisfying $E_{d}(\rho) \leq \lambda$ by

$$
\operatorname{tr}\left[\rho A^{\dagger} \otimes B^{\dagger}\left(\lambda \mathbb{I}-\phi_{d}\right) A \otimes B\right] \geq 0 \quad \forall A, B,
$$

where $\mathbb{I}$ is the $d^{2}$-dimensional identity matrix. We are interested in the intersection of the two cones (7) and (10):

$$
\mathcal{C}=\left\{\rho: \rho \succeq 0, E_{d}(\rho) \leq \lambda\right\}
$$


which is also a convex cone. Following [16], the dual cone of $\mathcal{C}$ is

$$
\mathcal{C}^{*}=\{X: \operatorname{tr}[\rho X] \geq 0 \forall \rho \in \mathcal{C}\} .
$$

A version of Farkas Lemma [17] states that each matrix $X \in \mathcal{C}^{*}$ can be written as

$$
X=\sum_{k} A_{k}^{\dagger} \otimes B_{k}^{\dagger}\left(\lambda \mathbb{I}-\phi_{d}\right) A_{k} \otimes B_{k}+\sum_{s} P_{s},
$$

where the two kinds of terms are of the form specified in (7) and (10).

Let us concentrate on the condition $E_{d}(\rho \otimes \sigma)>\lambda$. Instead of computing the supremum in (9) we consider a particular filtering operation $\tilde{A} \otimes \tilde{B}$, with which we obtain a lower bound on $E_{d}(\rho \otimes \sigma)$. The chosen form of $\tilde{A}$ and $\tilde{B}$ is

$$
\tilde{A}=\left\langle\phi_{A_{1} A_{2}}\right| \otimes \mathbb{I}_{A_{3}}, \quad \tilde{B}=\left\langle\phi_{B_{1} B_{2}}\right| \otimes \mathbb{I}_{B_{3}},
$$

where $\left|\phi_{A_{1} A_{2}}\right\rangle$ is the maximally entangled state between the systems corresponding to $\mathcal{H}_{A_{1}}$ and $\mathcal{H}_{A_{2}}$ (which have the same dimension), and $\mathbb{I}_{A_{3}}$ is the identity matrix acting on $\mathcal{H}_{A_{3}}$. Analogously for $\tilde{B}$. With a little calculation one obtains the matrix equality

$$
\operatorname{tr}_{12}\left[\tilde{A} \otimes \tilde{B}\left(\rho_{23} \otimes \sigma_{1}\right) \tilde{A}^{\dagger} \otimes \tilde{B}^{\dagger}\right]=z \operatorname{tr}_{2}\left[\rho_{23} \sigma_{2}^{\mathrm{T}}\right],
$$

where $z$ is a positive number, $\sigma^{\mathrm{T}}$ stands for the transpose of $\sigma$, and the subindexes indicate on which Hilbert spaces every matrix acts and where the partial traces are performed. Using (15), a sufficient condition for $E_{d}(\rho \otimes \sigma)>\lambda$ is

$$
\operatorname{tr}\left[\rho_{23}\left(\sigma_{2}^{\mathrm{T}} \otimes\left(\lambda \mathbb{I}-\phi_{d}\right)_{3}\right)\right]<0 .
$$

Let us show that there always exists a $\rho \in \mathcal{C}$ satisfying this inequality by creating a contradiction.

Suppose that no single $\rho \in \mathcal{C}$ satisfies (16), this is equivalent to saying that for all $\rho \in \mathcal{C}$

$$
\operatorname{tr}\left[\rho\left(\sigma^{\mathrm{T}} \otimes\left(\lambda \mathbb{I}-\phi_{d}\right)\right)\right] \geq 0 .
$$

Then, by definition (12), the matrix $\sigma^{\mathrm{T}} \otimes\left(\lambda \mathbb{I}-\phi_{d}\right)$ belongs to $\mathcal{C}^{*}$, and we can express it as in (13). One way of writing this is

$$
\sigma^{\mathrm{T}} \otimes\left(\lambda \mathbb{I}-\phi_{d}\right)-\Lambda\left(\lambda \mathbb{I}-\phi_{d}\right) \succeq 0,
$$

where $\Lambda$ is separable and maps matrices acting on $\mathbb{C}^{d} \otimes \mathbb{C}^{d}$ to matrices acting on $\mathcal{H}_{A_{1}} \otimes \mathcal{H}_{B_{1}} \otimes \mathbb{C}^{d} \otimes \mathbb{C}^{d}$. The partial trace of (18) over the space $\mathcal{H}_{A_{1}} \otimes \mathcal{H}_{B_{1}}$ is like (20), where $\$$ is a separable map. Then, Lemma 1 implies that the left-hand side of (18) is traceless, and a positive traceless matrix can only by the null matrix. Therefore $\Lambda\left(\lambda \mathbb{I}-\phi_{d}\right)=\sigma^{\mathrm{T}} \otimes\left(\lambda \mathbb{I}-\phi_{d}\right)$, whose partial transposition is

$$
\Lambda^{\Gamma}(\omega)=\sigma^{\mathrm{T \Gamma}} \otimes \omega,
$$

where we have used the following notation. If $\nu$ is a bipartite state, $\nu^{\Gamma}$ stands for its partial transposition [18], and $\Lambda^{\Gamma}$ is defined as $\Lambda^{\Gamma}(\nu)=\left[\Lambda\left(\nu^{\Gamma}\right)\right]^{\Gamma}$ for all $\nu$. The map $\Lambda^{\Gamma}$ in (19) is completely positive and separable, hence the matrix $\sigma^{\mathrm{T}}$ has positive partial transposition [18]. In Lemma 2 it is shown that a matrix $\sigma^{\mathrm{T \Gamma}}$ satisfying (19) must be separable. But this is in contradiction with the initial assumption that $\sigma$ is entangled. Therefore, the supposition that no single $\rho \in \mathcal{C}$ satisfies (16) is false.

Lemma 1. If $\$$ is a separable map (4) and $\lambda \in$ $[1 / d, 1)$ then

$$
\left(\lambda \mathbb{I}-\phi_{d}\right)-\$\left(\lambda \mathbb{I}-\phi_{d}\right) \succeq 0
$$

implies

$$
\operatorname{tr}\left[\left(\lambda \mathbb{I}-\phi_{d}\right)-\$\left(\lambda \mathbb{I}-\phi_{d}\right)\right]=0 .
$$

Proof. Werner states [2] are defined as

$$
\omega=\mu \omega^{-}+(1-\mu) \omega^{+} \quad \mu \in[0,1],
$$

where $\omega^{-}$and $\omega^{+}$are unit-trace matrices proportional, respectively, to the antisymmetric and symmetric projectors acting on $\mathbb{C}^{d} \otimes \mathbb{C}^{d}$. One can check that

$$
\omega^{\Gamma} \succeq 0 \quad \Leftrightarrow \quad \mu \leq 1 / 2 .
$$

Using notation defined above we can write (20) as

$$
\left[\omega-\$^{\Gamma}(\omega)\right]^{\Gamma} \succeq 0,
$$

where $\omega \propto\left(\lambda \mathbb{I}-\phi_{d}\right)^{\Gamma}$ is a normalized Werner state (22). Due to the fact that $\lambda<1$, the state $\omega$ in (24) does not have a positive partial transpose [18]. The depolarization map is defined as

$$
\Delta(\rho)=\int_{S U(d)} d U(U \otimes U) \rho(U \otimes U)^{\dagger},
$$

where $d U$ represents the invariant measure on the group $S U(d)$. The map $\Delta$ is completely-positive, separable, it always outputs a Werner state, and leaves Werner states invariant [2]. The twirl map is defined as $\Delta^{\Gamma}$, and it is also completely-positive and separable [15]. Then, we can apply $\Delta^{\Gamma}$ to the left-hand side of (24) obtaining the inequality

$$
\left[\omega-\tau \omega^{\prime}\right]^{\Gamma} \succeq 0,
$$

where $\tau \omega^{\prime}=\left[\Delta \circ \$^{\Gamma}\right](\omega)$, and $\tau \geq 0$ is a normalization factor. By the properties of $\Delta, \omega^{\prime}$ is a Werner state, and can be written as $\omega^{\prime}=\mu^{\prime} \omega^{-}+\left(1-\mu^{\prime}\right) \omega^{+}$. It is proven in [19] that the entanglement of Werner states, that is $\mu$, cannot be increased by SLOCC, therefore

$$
\mu^{\prime} \leq \mu
$$


Because the trace is invariant under partial transposition, from (26) it follows

$$
1-\tau \geq 0 .
$$

The left-hand side of (26) is the partial transposition of an unnormalized Werner state. Applying condition (23) to (26) we get

$$
\mu-\tau \mu^{\prime} \leq(1-\mu)-\tau\left(1-\mu^{\prime}\right) .
$$

The simultaneous satisfiability of (27), (28) and (29) is possible only if $\tau=1$. This implies that the trace of the left-hand side of (26) is zero, which is equivalent to $(21)$.

Lemma 2. If $\omega$ is an entangled Werner state and $\Lambda$ is a separable map such that $\Lambda(\omega)=\eta \otimes \omega$, then $\eta$ must be a separable state.

Proof. Let us separately prove the cases $\omega=\omega^{-}$ and $\omega \neq \omega^{-}$, where $\omega^{-}$is the antisymmetric state.

Firstly consider $\omega=\omega^{-}$. Two parties sharing the state $\omega^{-}$can, by LOCC, obtain a singlet $\phi_{2}$ with some probability. In particular, $A \otimes B \omega^{-} A^{\dagger} \otimes B^{\dagger} \propto \phi_{2}$ when $A=B=|1\rangle\langle 1|+| 2\rangle\langle 2|$. This implies that there exists a SLOCC transformation $\Lambda^{\prime}$ such that $\Lambda^{\prime}\left(\omega^{-}\right)=\eta \otimes$ $\phi_{2}$. Consider the Schmidt number for density matrices $K$, defined in [20]. If $\eta$ is an entangled state then $K\left(\eta \otimes \phi_{2}\right)>2$. One can also check that $K\left(\omega^{-}\right)=$ 2. But this is in contradiction with the fact that the Schmidt number $K$ is non-increasing under SLOCC transformations. Therefore $\eta$ must be separable.

Secondly consider $\omega \neq \omega^{-}$. Because the map $\Delta$ leaves Werner states invariant we can write

$$
\left[\left(\mathbb{I}_{1} \otimes \Delta_{2}\right) \circ \Lambda \circ \Delta\right](\omega)=\eta_{1} \otimes \omega_{2},
$$

where subindexes denote the system on which each map acts. It is proven in [19] that if $\$$ is a separable map and $\omega$ is an entangled Werner state (23) satisfying $[\Delta \circ \$ \circ \Delta](\omega)=\omega$, then $\Delta \circ \$ \circ \Delta=\Delta$. Using this fact, one can define the states $\eta^{-}$and $\eta^{+}$as

$$
[(\mathbb{I} \otimes \Delta) \circ \Lambda \circ \Delta]\left(\omega^{ \pm}\right)=\eta^{ \pm} \otimes \omega^{ \pm} .
$$

Because $\omega^{+}$and the compound map are separable, $\eta^{+}$must be separable too. Apart from this $\eta^{ \pm}$are arbitrary. Using representation (22) and linearity we have

$$
\eta \otimes \omega=\mu \eta^{-} \otimes \omega^{-}+(1-\mu) \eta^{+} \otimes \omega^{+} .
$$

Consider both sides of this equality as a vectors in the space of hermitian matrices. Because the left-hand side is a product vector, so must be the right-hand side. The fact that $\omega^{+}$and $\omega^{-}$are orthogonal implies that $\eta^{-}=\eta^{+}$, and then $\eta=\eta^{+}$is separable.

Final remarks. The method used to prove the theorem does not say much about the state $\rho$, whose entanglement is enhanced by $\sigma$. But clearly, if the state $\sigma$ has a positive partial transpose (PPT), whatever the values of $d$ and $\lambda$, the corresponding state $\rho$ is not PPT. An other fact about $\rho$ is that it is related to an entanglement witness [10] that detects $\sigma$. Therefore, the problem of finding $\rho$ given $\sigma$ is at least as hard as finding an entanglement witness that detects $\sigma$. More concretely, the operator

$$
W=\operatorname{tr}_{2}\left[\rho_{12}\left(\lambda \mathbb{I}-\phi_{d}\right)_{2}\right]
$$

can be proved to be an entanglement witness by imposing $E_{d}(\rho) \leq \lambda$. That $W$ detects $\sigma$ follows from inequality (16). As a consequence of the theorem, the set of witnesses of the form (33) is complete, in the sense that it detects all entangled states.

A state $\rho$ is said to be 1 -distillable if $E_{2}(\rho)>1 / 2$. It is known that all 1-distillable states are distillable [15]. It is proven in [10] that, for each bipartite state $\rho$ not being PPT nor 1-distillable, there exists a PPT state $\sigma$, such that $\rho \otimes \sigma$ is 1-distillable. One can obtain a kind of dual result as a corollary of the theorem shown above. That is, for each PPT state $\sigma$, there exists a state not being 1-distillable $\rho$, such that $\rho \otimes \sigma$ is 1-distillable.

Concluding, the theorem proven in this paper clarifies some aspects of entanglement theory that remained obscure before. In particular, whether there is a way in which the entanglement present in bound entangled states can manifest itself. With this new insight it becomes clear that, though strong irreversible processes take place in the preparation of bound entangled states, the pure-state entanglement is still there, and can be used in some sense. This shows that all quantum correlations have a distinctive behavior, and thus, give an advantage over classical correlations.

Acknowledgments. The author is thankful to Andrew Doherty, Nick Jones, Barbara Kraus, Yeong Cherng Liang, Guifré Vidal and Karl Vollbrecht for discussions and suggestions on the manuscript. This work has been supported by the U.K. EPSRC's "IRC QIP".

[1] A state $\rho$ acting on the Hilbert space $\mathcal{H}_{A} \otimes \mathcal{H}_{B}$ is said to be separable if it can be written as

$$
\rho=\sum_{k} \alpha_{k} \otimes \beta_{k},
$$

where $\alpha_{k}$ and $\beta_{k}$ are positive matrices acting respectively on $\mathcal{H}_{A}$ and $\mathcal{H}_{B}[2]$.

[2] R. F. Werner; Phys. Rev. A 40, 4277 (1989).

[3] C. Bennett, G. Brassard, C. Crepeau, R. Jozsa, A. Peres, W. K. Wootters; Phys. Rev. Lett. 70, 1895 (1993). 
[4] C. Bouwmeester, J. W. Pan, K. Mattle, M. Elbl, H. Weinfurter, A. Zeilinger; Nature (London) 390, 575 (1997).

[5] T. Mor; quant-ph/9608005.

[6] J. Barrett; Phys. Rev. A, 65, 042302 (2002).

[7] M. Horodecki, P. Horodecki, R. Horodecki; Phys. Rev. Lett., 80, no. 24, pp. 5239 (1998).

[8] M. Horodecki, P. Horodecki, R. Horodecki; quant$\mathrm{ph} / 9807091$.

[9] K. Horodecki, M. Horodecki, P. Horodecki, J. Oppenheim; Phys. Rev. Lett., vol. 94, no. 16, 160502 (2005).

[10] B. Kraus, M. Lewenstein, J. I. Cirac; Phys. Rev. A 65, 042327 (2002).

[11] P. W. Shor, J. A. Smolin, A. V. Thapliyal; quant$\mathrm{ph} / 0005117$.

[12] P. Horodecki, M. Horodecki, R. Horodecki; Phys. Rev. Lett. 82, 1056 (1999).
[13] M. A. Nielsen, I. L. Chuang; Quantum computation and quantum information (Cambridge University Press, Cambridge, 2000).

[14] G. Vidal; J.Mod.Opt. 47, 355 (2000).

[15] M. Horodecki, P. Horodecki; Phys. Rev. A. 59, 4206 (1999).

[16] S. Boyd, L. Vandenberghe; Convex Optimization (Cambridge University Press, Cambridge 2000).

[17] B. D. Craven, J. J. Koliha; SIAM J. Math. Anal. 8, 983 (1977).

[18] M. Horodecki, P. Horodecki, R. Horodecki; Phis. Lett. A 223, 1 (1996).

[19] H. Yuang, Ll. Masanes; In preparation.

[20] B. M. Terhal, P. Horodecki; Phys. Rev. A Rapid Communications 61, 040301 (2000). 